\title{
Lithium in Metal Deficient K Giant Stars: The Absence of Dust Signature
}

\author{
Ramiro de la Reza ${ }^{1}$, Licio da Silva ${ }^{1}$, Natalia A. Drake ${ }^{1,2}$ and Marco A. \\ Terra $^{3}$ \\ ${ }^{1}$ Observatório Nacional - Rio de Janeiro - 20921-400 Brazil \\ ${ }^{2}$ Astronomical Institute - St. Petersburg University - St. Petersburg - \\ 198904 Russia
}

${ }^{3}$ Observatório do Valongo - UFRJ - Rio de Janeiro - 20080-090 Brazil

\begin{abstract}
The ${ }^{7} \mathrm{Li}$ enrichment - mass loss scenario has been constructed using the strong connection found between solar metallicity Li-rich giants and IRAS sources. We show here that the Li-rich metal poor first-ascent red giants are not IRAS sources, although they are loosing Li enriched matter in form of gas.
\end{abstract}

\section{The prompt ${ }^{7} \mathrm{Li}$ enrichment - mass loss scenario}

By this scenario, suggested by de la Reza et al. (1996), all low mass giants $\left(M<2.5 M_{\odot}\right)$ suffer in the upper part of the red giant branch (RGB) a rapid ${ }^{7} \mathrm{Li}$ enrichment process of internal origin producing the formation of a Li-enriched circumstellar shell (CS) of gas and dust. This last one produces a signature in the IRAS colors. The CS detaches from the star transporting this way the new ${ }^{7} \mathrm{Li}$ into the interstellar medium. The fresh ${ }^{7} \mathrm{Li}$ remaining in the stars' photospheres is after depleted by convection. All these processes can be followed by means of closed loops (representing the gradually disappearance of the CS) in an IRAS diagram.

Smith (1998), collecting IRAS fluxes of metal deficient stars, concludes that mass loss in giants appears to be low due to the lack of observed IRAS fluxes. This is true for dust mass loss but not necessarily for the gas mass loss. Poor metal giants do not have sufficient IRAS fluxes because they do not have sufficient metals to produce enough dust. In fact, the dust-to-gas ratio, $\psi$, depends mainly on the abundances of metal dust producers and the drift velocity between gas and dust. If we consider the drift velocity as zero and the dust producers measured by the iron abundance, we then have $\psi \sim[\mathrm{Fe} / \mathrm{H}]$. We can calculate the evolution of the CS in an IRAS diagram which is presented in the figure together with observed points corresponding to some Li-rich mild deficient (labeled by filled symbols) and Li-poor giants (open symbols; having fluxes limits). Recently spectra of the Li depleted giant HD 68298 have been obtained with FEROS at La Silla, Chile, under the Observatório Nacional-ESO agreement. From them, we determined for this star:

$T_{\text {eff }}=4000 \mathrm{~K}, \log g=0.75, \log \epsilon_{\mathrm{Li}}=0.2$ and $[\mathrm{Fe} / \mathrm{H}]=-0.4$ 


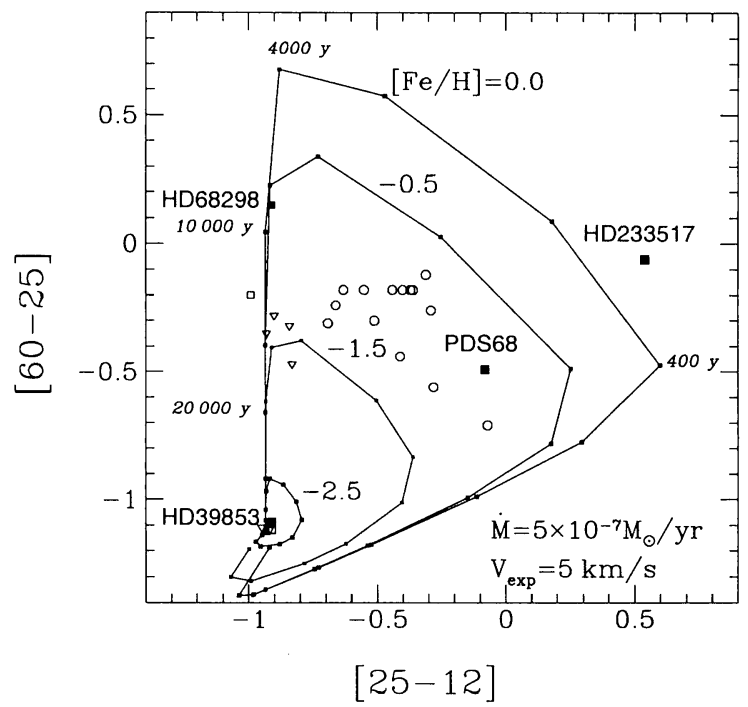

\section{The scenario for deficient stars}

The dependence of the dust optical depth, $\tau_{d u s t}$, on the density of dust particles is given by $\tau_{d u s t} \sim \psi \cdot \dot{M}$, where $\dot{M}$ is the gas mass loss (de la Reza et al. 1996). If few IRAS fluxes are measured among the metal poor giants, this is because $\tau_{\text {dust }}$ has low values corresponding to the low values of $\psi$ and not necessary to the low values of $\dot{M}$. The curves in the figure are calculated for $\dot{M}=5 \times 10^{-7} M_{\odot} / \mathrm{yr}$. IRAS fluxes are observed only for mild metal deficient giants $([\mathrm{Fe} / \mathrm{H}]>-1.0)$. The small loops corresponding to very deficient giants explain why no IRAS fluxes are observed for these stars. Mass loss in very Li-rich and very metal poor giants must be put in evidence by other methods as detecting asymmetries in $\mathrm{Na}$ resonance lines.

As an example the very Li-rich RGB star discovered recently in the globular cluster $\mathrm{M} 3([\mathrm{Fe} / \mathrm{H}]=-1.5)$ by Kraft et al. 1999 is very probably suffering a mass loss but is supposed not to present an important dust signature. If evolving giants are the main source of dust in M3, few dust must be present in this globular cluster. Penny et al. (1997) have found almost no dust in the central $14^{\prime \prime}$ region of $\mathrm{M} 3$.

Acknowledgments. R. de la R. and L. da S. thank CNPq for grants 301375/ 86-0 and 200580/97-3 respectively. N.A.D. and M.A.T. thank FAPERJ for the financial support under the contracts E-26/151.172/98 and E-26/150.571/98 respectively.

\section{References}

de la Reza, R., Drake, N.A., \& da Silva, L. 1996, ApJ, 456, L115

Kraft, R.P., Peterson, R.C., Guhathakurta, P., Sneden, C., Fulbright, J.P., \& Langer, G.E. 1999, ApJ, 518, L53

Penny, A.J., Evans, A., \& Odenkirchen, M. 1997, A\&A, 317, 694

Smith, G.H. 1998, PASP, 110, 1119 